\title{
Research on super capacitance properties of modified activated carbon in sodium sulfate electrolyte
}

\author{
Wang Lin ${ }^{1,2, a}$, Wang Chun ${ }^{1}$, LiuYing ${ }^{3}$, Yan Heng ${ }^{1}$, Zhang Xiangbo ${ }^{1}$ and Xu Hongfeng ${ }^{2}$ \\ ${ }^{1}$ School of Materials and Metallurgy, University of Science and Technology Liaoning, Anshan114051,China \\ ${ }^{2}$ School of Materials Science and Engineering, Dalian Jiaotong University, Dalian, 116028,China \\ ${ }^{3}$ Tangshan vocational college of science and technology, Tangshan,063000, China
}

\begin{abstract}
In order to improve the specific capacity and energy density of activated carbon electrode materials, activated carbon was modified by surface treatment using hydrogen peroxide. The modified carbons were characterized the changes of pore structure and functional groups before and after modification by $\mathrm{N}_{2}$ adsorption-desorption isotherms and FTIR spectroscopy. The three electrode system was assembled with modified activated carbon as electrode materials and $\mathrm{Na}_{2} \mathrm{SO}_{4}$ as the electrolyte. The influence of surface modification on the performance of EDLCs was studied by galvanostatic charge-discharge, cyclic voltammetry, and alternating current impedance. The results show that the surface chemistry of the modified activated carbon material has changed, the specific surface area has changed and the pore size has not changed much with the introduction of new oxygen-containing functional groups. The specific capacity of the activated carbon material modified by $5 \mathrm{~h}$ is $75 \mathrm{~F} / \mathrm{g}$, which is $86.1 \%$ higher than that before the modification. It can be seen that the change of functional group after the modification of activated carbon can help to increase the specific capacity.
\end{abstract}

\section{Introduction}

Supercapacitors have received wide attention as an efficient, environmentally friendly, and renewable energy device. The traditional capacitor has low power density, short cycle life, slow charge and discharge speed, which makes the application market shrink compared with supercapacitors. Electrolyte and electrode materials are important components of supercapacitors and critical to the performance of capacitors ${ }^{[1-3]}$. As an important electrode material, activated carbon material has the advantages of larger specific surface, excellent thermal, electrical conductivity, good chemical corrosion resistance, small thermal expansion coefficient, low cost, easy availability and easy industrialization ${ }^{[4-8]}$. The pore structure and surface chemistry of carbon materials have an important influence on the formation of electric double layers $^{[9-13]}$.

In this paper, the activated carbon was modified with $30 \% \mathrm{H}_{2} \mathrm{O}_{2}$. The modified activated carbon was used as the electrode material of the supercapacitor. Physical characterization was carried out by Fourier transform infrared spectroscopy and $\mathrm{N}_{2}$ adsorption-desorption method. Activated carbon was used as the electrode material. The cyclic voltammetry, galvanostatic charge and discharge characteristics of activated carbon with different modification time were measured in $1 \mathrm{~mol} / \mathrm{L}$

a Corresponding author: E-mail:wanglin6051@163.com 
$\mathrm{Na}_{2} \mathrm{SO}_{4}$ electrolyte, the specific capacity of the activated carbon was calculated and the effects of different modification conditions on the capacitance properties of carbon materials were investigated.

\section{Experimental}

\subsection{Materials and reagents}

activated carbon (AC), PTFE emulsion (PTFE), acetylene black, foam nickel, $30 \% \mathrm{H}_{2} \mathrm{O}_{2}, \mathrm{Na}_{2} \mathrm{SO}_{4}$, absolute ethanol, etc. All reagents are analytical grade.

\subsection{Activated carbon modification}

The activated carbon for the study was prepared by SUNRISE POWER. A carbon (AC-0.5-AC-6) was prepared through oxidation of $\mathrm{AC}(1 \mathrm{~g})$ with $50 \mathrm{~mL}$ of 35 wt $\% \mathrm{H}_{2} \mathrm{O}_{2}$ solution under stirring for $0.5 \mathrm{~h}-6 \mathrm{~h}$ at room temperature. Recorded as $\mathrm{AC}-0.5, \mathrm{AC}-1, \mathrm{AC}-2, \mathrm{AC}-3$, $\mathrm{AC}-4, \mathrm{AC}-5$ and $\mathrm{AC}-6$, untreated activated carbon is recorded as AC-0.The resultant material was extensively rinsed with distilled water. Then they were dried in a vacuum drying oven.

\subsection{Performance characterization}

\subsubsection{Fourier transform infrared spectrometer (FTIR)}

FTIR was carried out by $\mathrm{KBr}$ tableting method. The appropriate $\mathrm{KBr}$ and activated carbon before and after modification were mixed, grinded and tableted. The TENSOR 27 Fourier infrared spectrometer was used to analyze the changes in surface functional groups of carbon materials, with wavelength range of $250 \sim 4000 \mathrm{~cm}^{-1}$.

\subsubsection{Nitrogen absorption and desorption experiment}

The nitrogen $\left(\mathrm{N}_{2}\right)$ isotherm adsorption/desorption test is one of the methods for testing parameters such as specific surface area and pore size distribution of materials. The specific surface area and pore distribution of the material were tested using a Micrometeritics ASAP 2000 specific surface area tester, vacuum pretreatment at 393K, absorption/desorption at liquid nitrogen temperature $\left(-196^{\circ} \mathrm{C}\right)$, and data was recorded and analysed by computer.

\subsubsection{Electrochemical characterization}

Electrodes $(1 \mathrm{~cm}$ diameter $)$ were pressed from a mixture of the studied carbons (80 wt $\%), \operatorname{PTFE}(10 \mathrm{wt} \%)$, carbon black (10 wt \%). Three-electrode with $\mathrm{Hg} / \mathrm{Hg}_{2} \mathrm{SO}_{4}$ as a reference electrode comprised the studied carbon and Pt electrode as working and counter electrodes, respectively. Cyclic voltammetry and galvanostatic charge/discharge cycling were measured by a CHI660D electrochemical workstation in $1 \mathrm{~mol} / \mathrm{L} \mathrm{Na}_{2} \mathrm{SO}_{4}$ electrolyte solution. The specific capacitance $(C s)$ can be calculated according to the following equation:

$$
C s=I t / m U
$$

where in $I(\mathrm{~A}), t(\mathrm{~s}), m(\mathrm{~g})$ and $U(\mathrm{~V})$ represent the discharge current, discharge time, mass of active material and window potential, respectively.

\section{Results and discussion}

\subsection{Infrared spectroscopy}

In order to investigate the Changes in oxygen-containing functional groups on the surface of activated carbon. Fourier transform infrared (FTIR) spectroscopy was conducted. The result is shown in Figure 1.

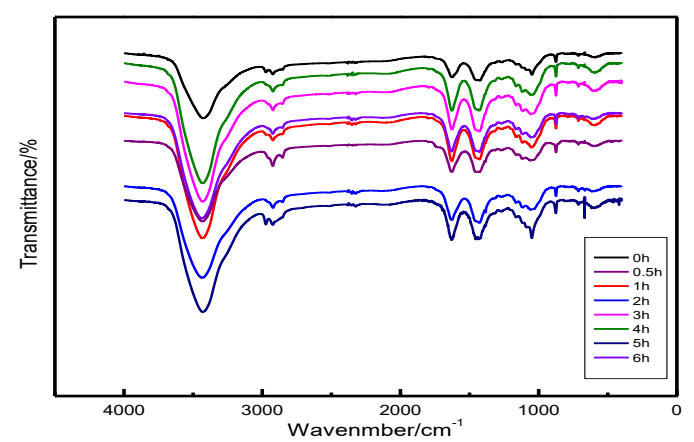

Figure 1. FTIR spectra of activated carbons before and after modification

Figure 1 is a FTIR spectrum of a carbon material before and after surface modification. It can be seen from the figure that after $\mathrm{H}_{2} \mathrm{O}_{2}$ modification treatment, the -COO-anti-extension peak of AC-0 at $1570 \mathrm{~cm}^{-1}$ 
disappears after modification, and the -OH stretching vibration peak of the carbon material modified by $\mathrm{H}_{2} \mathrm{O}_{2}$ is obviously enhanced at $3430 \mathrm{~cm}^{-1}$, especially the AC enhancement with the modification time of $5 \mathrm{~h}$ is most obvious. The $\mathrm{C}-\mathrm{O}$ stretching vibration and $\mathrm{O}-\mathrm{H}$ out-of-plane bending occurred at $1264 \mathrm{~cm}^{-1}$ and $669 \mathrm{~cm}^{-1}$ respectively, and the carbonyl stretching vibration peak appeared at $1640 \mathrm{~cm}^{-1}$, indicating that the modified CO content may increase, and the surface of the carbon material was introduced with $-\mathrm{OH}{ }^{[14-18]}$. It can be seen that the surface modification has a great influence on the surface chemistry of the carbon material. Through the modification of hydrogen peroxide, some functional groups and groups are introduced on the surface of the activated carbon, which is beneficial to improve the capacitance performance of the activated carbon electrode.

\section{2 $\mathrm{N}_{2}$ adsorption-desorption isotherm}

Fig.2 is nitrogen adsorption-desorption isotherms of activated carbons. It can be seen from the figure that the activated carbon which was modified that the adsorption amount of $\mathrm{N}_{2}$ was changed at different extents, but the shape of the adsorption curve and the desorption curve did not change substantially. All adsorption isotherms are classic V-type isotherms. The adsorption capacity in the low pressure is very low and slowly increases. The relative pressure at 0.9 is a significant lag in the desorption curve. According to the trend of the adsorption isotherm and the hysteresis loop, it can be seen that all activated carbon is a mesoporous or microporous material.

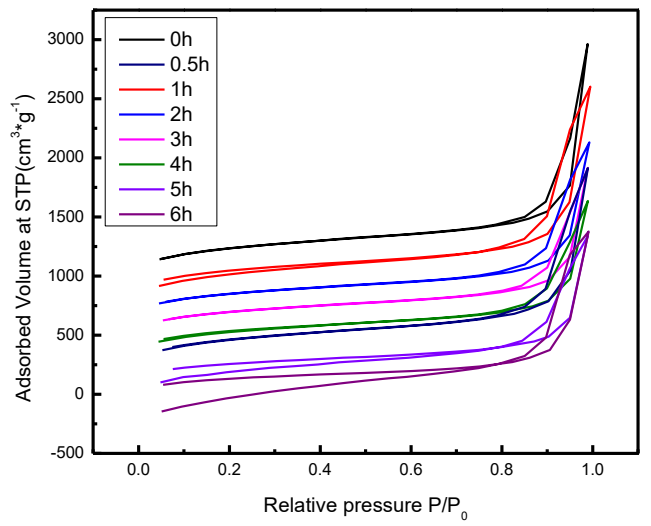

Figure 2. Nitrogen adsorption-desorption isotherms of activated carbons
The changes in specific surface area and pore structure parameters of carbon materials before and after modification are displayd in table 1 . The modification treatment did not change in the pore structure of the micropores and mesopores of the activated carbon, and the sample still retained the characteristics of the microporous material. However, the specific surface area of the carbon material are decreased at different modification times. The total pore volume and micropore volume of carbon materials are reduced.The average pore size of the modified carbon material is decreased slightly. The results of different modification time modification treatments, except for a certain decrease in specific surface area, other pore structure parameters did not change much.

Table1. Specific surface area and pore diameter of active

\begin{tabular}{c|c}
\multicolumn{2}{c}{ carbon } \\
\hline $\mathrm{S}_{\text {BET }}\left(\mathrm{m}^{2} / \mathrm{g}\right)$ & $\mathrm{D}(\mathrm{nm})$ \\
\hline 1593.241 & 3.345 \\
1513.394 & 3.335 \\
1458.420 & 3.365 \\
1313.478 & 3.366 \\
1296.835 & 3.354 \\
1261.308 & 3.376 \\
1143.590 & 3.360 \\
1069.074 & 3.036 \\
\hline
\end{tabular}

\subsection{Electrochemical properties}

To compare the electrochemical properties of carbon materials before and after modification. $\mathrm{CV}$ and galvanostatic charge-discharge measurements were performed. $\mathrm{CV}$ curves of $\mathrm{AC}-5$ obtained at different scan rates and $\mathrm{CV}$ curves obtained at a scan rate of $20 \mathrm{mV} / \mathrm{s}$ for different treatment time are shown in Fig. 3 and Fig.4.

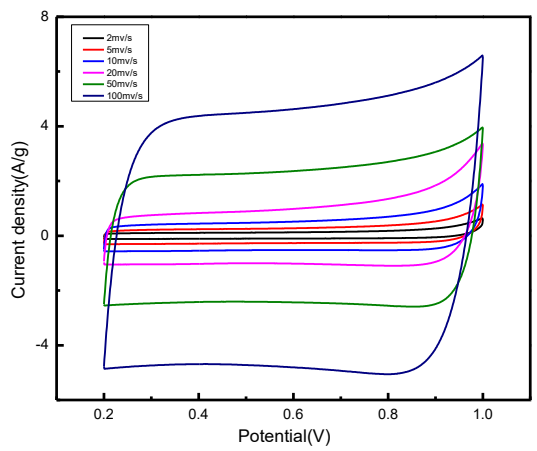

Figure 3. AC-5 CV curves obtained at different scan rates 


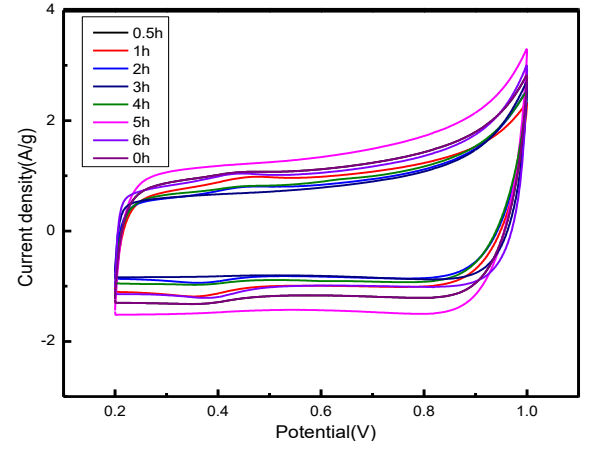

Figure 4. AC-5 CV curves obtained at a scan rate of $20 \mathrm{mV} / \mathrm{s}$ for different treatment time

It can be seen from Fig. 3 and Fig. 4 that the cyclic voltammetry curves all show an approximately rectangular shape and do not contain obvious redox peaks, indicating that electrostatic physical adsorption is the main energy storage method for electrode materials. However, due to the existence of a large number of micropores in the activated carbon, the electrolyte infiltration and the positive and negative ions penetrate the pores in different ways, resulting in different equivalent series resistance (ESR), and the cyclic voltammetry deviates from the ideal rectangular shape to some extent. As the scanning speed increases, the response current increases proportionally, indicating that the electrolyte ions migrate faster and have less resistance in the activated carbon tunnel. The cyclic voltammetry curve is basically rectangular, indicating good reversibility.

The galvanostatic charge/discharge characteristics of AC-0-AC-6 in 1mol/L Na $\mathrm{Na}_{4}$ were recorded in Fig.5.

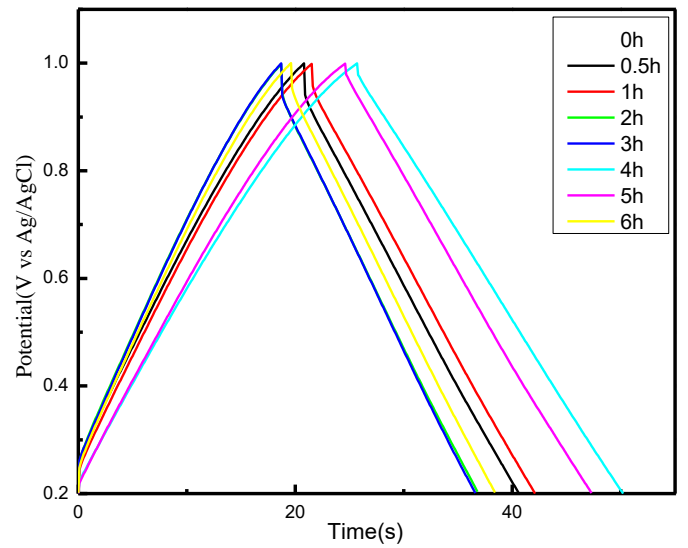

Figure 5. The charge-discharge curves obtained at $2 \mathrm{~A} / \mathrm{g}$ for sample AC-0-AC-6 in $1 \mathrm{~mol} / \mathrm{L} \mathrm{Na}_{2} \mathrm{SO}_{4}$

It can be seen from Fig. 5 that the charge-discharge curve has a relatively symmetrical isosceles triangle shape, indicating that the capacitor has good capacitance characteristics, and at the moment of charging and discharging, the potential drop is small, indicating that the internal resistance of the activated carbon electrode is small. Accroding to the charge-discharge curve, the specific capacitance value of the activated carbon electrode can be calculated. Fig.6 shows the specific capacitance of different samples calculated from their discharge processes at different current densities.

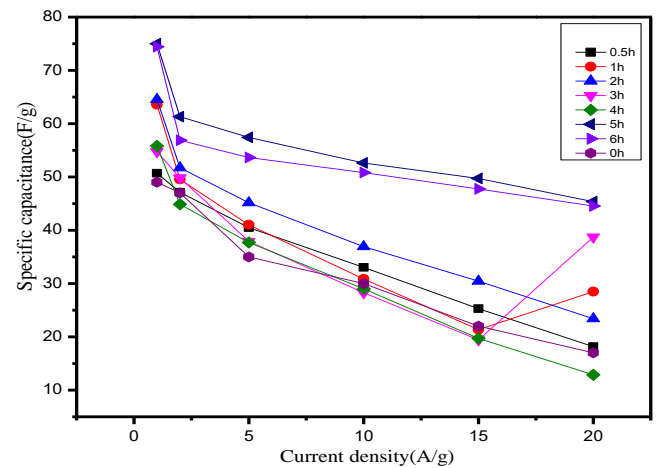

Figure 6. The specific capacitance of AC-0-AC-6 at different current densities

It can be seen from Fig.6 that the variation rules of activated carbon at different modification times are not much different. Before the modification time $5 \mathrm{~h}$, the specific capacity gradually increased, and reached the maximum at the modification time of $5 \mathrm{~h}$. When the modification time is $6 \mathrm{~h}$, the specific capacity does not continue to increase, it is slightly smaller than the modification time of $5 \mathrm{~h}$, indicating that the electrochemical performance of the activated carbon is the best at $5 \mathrm{~h}$.

\section{Conclusion}

(1)The modification treatment leads to large changes in the surface chemistry of the carbon, and the original -COO- content is reduced. Functional group $=\mathrm{CO}$ and $-\mathrm{OH}$ on the carbon surface are increased. the specific capacity of the carbon materials are increased, thereby improving the electrochemical performance of the electrode.

(2)By comparing the relationship between capacitance and structure of activated carbon materials with different modification times, due to the pore structure, surface chemistry and wettability of carbon materials, with the increasing of the specific surface area, the specific 
capacitance is not increasing always. There is no direct correlation between the specific capacity of activated carbon materials and the change in specific surface area.

(3)It have been shown by both galvanostatic charge/discharge and cyclic voltammetry experiment that the surface functional groups introduced by the modification of carbon materials can effectively increase their specific capacity. By comparing the activated carbon electrode materials with different modification time, the specific capacity of activated carbon with the modification time of $5 \mathrm{~h}$ is the highest. When the current density is $1 \mathrm{~A} / \mathrm{g}$, the specific capacity is the largest, which is $75 \mathrm{~F} / \mathrm{g}$ compared to the modification time of $0 \mathrm{~h}$ (specific capacity $40.3 \mathrm{~F} / \mathrm{g}$ ) increased by $86.1 \%$, and it was found that the modification time was not as long as possible.

\section{Acknowledgement}

This work was supported by Provincial College Students Innovation and Entrepreneurship Training Program (NO. 201710146000029) , Youth fund by University of Science and Technology Liaoning (NO.2015QN27) and National College Students Innovation and Entrepreneurship Training Program(NO. 201810146004)

\section{Reference}

[1] J.P. Cheng, Q.L. Shou, J.S. Wu, F. Liu, V.P. Dravid, X.B. Zhang.J. Electroanal. Chem. 698 (2013)

[2]Yu A P, Davies, Chen Z W. Wiley-VCH,(2011)

[3] LIU Ya-Fei, HU Zhong-Hua*,XU Kun, ZHENG Xiang-Wei, GAO Qiang. Acta Phys. -Chim. Sin.(2008)

[4] Meier J C, Katsounaros I, Galeano C, etal. Energy Environ Sci.(2012)

[5] K. Fic, G. Lota, M. Meller and E. Frackowiak, Energy Environ. Sci.5, 5842-5850 (2012)

[6] Frackowiak E. Phys. Chem. (2007)

[7]Kotz R， Carlen.ElectrochimicaAct,45,2483(2000)

[8]Qu D Y. J. Power Sources, 109, 403-411(2002)

[9]Burke A. J.Power sources，91,37-50(2000)

[10]Sharma P. J.hydrogen energy:, 34,4889-4899(2009)

[11]Zhang Y. IJHE,34,4889-4899(2009)

[12]Hamon $\mathrm{M} \mathrm{A}, \mathrm{Hu} \mathrm{H}$, Bhowmik $\mathrm{P}$, etal.
Chem.Phys.Lett., 347, 8-12( 2001)

[13] Rosca I D, Watari F, Uo M, etal. Carbon, 43(2005)

[14]Qu D, Shi H. J.Power Sources 74,99-107(1998)

[15]Bichat H P, RAY.E.Carbon,48,4351-4361(2010)

[16]He X．J PHYS CHEM SOLIDS, 70,738-744(2009)

[17]J.P.Zheng. J.Power Sources， 62(1996)

[18]R.N.Reddy. J.Power Sources， 132(2004) 